\title{
Ionospheric and induced field leakage in geomagnetic field models, and derivation of candidate models for DGRF 1995 and DGRF 2000
}

\author{
Nils Olsen ${ }^{1}$, Frank Lowes ${ }^{2}$, and Terence J. Sabaka ${ }^{3}$ \\ ${ }^{1}$ Danish National Space Center, Juliane Maries Vej 30, DK-2100 Copenhagen, Denmark \\ ${ }^{2}$ Physics Department, University of Newcastle upon Tyne, U.K. \\ ${ }^{3}$ Geodynamics Branch, NASA GSFC, Greenbelt/MD, U.S.A.
}

(Received December 7, 2004; Revised June 24, 2005; Accepted June 28, 2005)

\begin{abstract}
As part of the 9th generation of the IGRF defined by IAGA, we proposed a candidate model for DGRF 1995 and two candidate models for DGRF 2000. These candidate models, the derivation of which is described in the present note, are based on the "Comprehensive Model, Version 4 (CM4)", and on the "Ørsted Main and Secular Variation Model (OSVM)"; two parent models that have been published elsewhere (Olsen, 2002; Sabaka et al., 2004; Lowes and Olsen, 2004). However, the main field part of OSVM is contaminated by "leakage" of the ionospheric field and its induced counterpart, which affects mainly the zonal coefficients $g_{1}^{0}, g_{3}^{0}, \ldots$, by $1-2 \mathrm{nT}$. We describe the reason for this contamination, and present a method to correct for it. Since not only OSVM, but probably all main field models that are derived primarily from data around local midnight suffer from this effect, the presented scheme can also be applied to approximately correct these models.

Key words: Geomagnetic Reference Model, IGRF/DGRF, ionospheric currents, induction, spherical harmonic analysis.
\end{abstract}

\section{Introduction}

The 9th generation of the IGRF (Mandea, 2005) is based on 6 candidate models for DGRF 1995, and on 5 candidate models for DGRF 2000. These candidate models have been submitted to IAGA working group V-MOD (formerly $\mathrm{V}-8$ ) in March 2003, and during the IAGA conference in Sapporo in July 2003 it was decided to take for DGRF 1995 and DGRF 2000 a straight mean of the coefficients of all submitted candidate models.

This note describes the derivation of three out of the 11 submitted candidate models: a candidate model for DGRF 1995 and two candidate models for DGRF 2000. They are based on two parent models: the "Comprehensive Model, Version 4 (CM4)" of Sabaka et al. (2004), and the "Ørsted Main and Secular Field Model (OSVM)" described in Olsen (2002) and Lowes and Olsen (2004). Special emphasis has been laid on minimizing model contamination by ionospheric and induced contributions. Although ionospheric field contributions at middle and low latitudes are heavily reduced during night-time hours (which is the reason for the usual selection of night-time data in geomagnetic field modeling), secondary currents induced in the Earth produce field contributions even during night when the primary, ionospheric, currents vanish. In addition, ionospheric currents at polar latitudes are almost always present, which makes their minimization by means of data selection difficult. Most, if not all, main field models that have been derived primarily using data around local midnight suffer

Copyright (c) The Society of Geomagnetism and Earth, Planetary and Space Sciences (SGEPSS); The Seismological Society of Japan; The Volcanological Society of Japan; The Geodetic Society of Japan; The Japanese Society for Planetary Sciences; TERRAPUB from this effect.

In following we will briefly discuss the parent models from which our candidate models are extracted, discuss the effect of "leakage" of ionospheric and induced fields in main field models, and propose a correction scheme.

\section{Candidate Models for DGRF 1995 and 2000 Based on the Comprehensive Model (CM)}

These candidate models are based on the main field part of the Comprehensive Model CM4 (Sabaka et al., 2004), which is an extended version of the model described in Sabaka et al. (2002). It is based on data from the satellites POGO (magnetic scalar data), Magsat (scalar+vector data), Ørsted (scalar + vector data) and CHAMP (scalar data) as well as on observatory hourly mean values spanning the years 1960 to 2000 . The model attempts to describe the major quiet-time contributions to the Earth's magnetic field (core and crustal fields, fields due to currents in the ionosphere and magnetosphere, and due to secondary, Earthinduced, currents) by co-estimation of all these sources. About 2.2 million data points have been used to estimate about 25,000 model parameters.

We will only give a short description of the model; the reader is referred to Sabaka et al. (2004) for more information. Software for computing magnetic field values and extraction of model coefficients can be found at http://core2.gsfc.nasa.gov/CM/.

The core and lithospheric fields are together expressed as the negative gradient of a potential function represented by a degree and order 65 internal spherical harmonic expansion, with secular variation represented by cubic B-splines through degree and order 13, using a knot spacing of $2.5 \mathrm{yrs}$. 
A set of static vector biases is solved for at each observatory, including its breaks. These biases represent smallscale lithospheric anomalies whose spatial frequencies are above the spherical harmonic truncation level.

Ionospheric field contributions are expressed as the negative gradient of a potential function which is expanded in harmonic functions in a quasi-dipole coordinate system that is aligned with the ambient magnetic field. Temporally, these functions are mainly sun-synchronous but are modulated with annual and semi-annual seasonal variability. Induced contributions are accounted for by using an a priori model of electrical conductivity of the mantle. Solar activity influence is introduced through an amplification factor, assumed to be equal for all ionospheric (and corresponding induced) coefficients, scaled by the $F_{10.7}$ solar radio flux values.

Magnetospheric field contributions are cast as the negative gradient of a potential represented by an external spherical harmonic expansion in dipole coordinates, which has regular daily and seasonal periodicities. Magnetospheric ring current variability is modeled as a linear function of the $D_{\text {st }}$ index for external dipole terms only. Induced contributions of the magnetosphere are treated in a similar manner as the ionosphere and are thus coupled with an internal expansion via the same a priori conductivity model.

Finally, currents coupling the ionosphere and magnetosphere are considered by a toroidal field contribution in quasi-dipole coordinates.

Our candidate for DGRF 1995 (called DGRF1995-CM) is a $n_{\max }=10$ truncation of the main-field part of CM4 at epoch 1995.0, rounded to the nearest nT. Our first candidate for DGRF 2000 (called DGRF2000-CM) is a $n_{\max }=13$ truncation of CM4 at epoch 2000.0, rounded to the nearest $0.1 \mathrm{nT}$.

\section{Candidate Model for DGRF 2000 Based on the Ørsted Main and Secular Field Model (OSVM) 3.1 Description of the OSVM}

Our second candidate for DGRF2000 is based on the main field part of the OSVM model (Olsen, 2002). This model is a spherical harmonic expansion of the static main field (up to degree/order 29) for epoch 2000 and of the secular variation (up to degree/order 13). It used Ørsted nighttime data (local time between 19:00 and 07:00) spanning more than two years (March 1999 to September 2001) and was derived applying new modeling approaches for a correct statistical treatment of data errors and for considering external field contributions. About 14,000 scalar data points at polar latitudes $\left(>50^{\circ}\right.$ dipole latitude) were used, together with about 30,000 scalar data points and about $24,600 \mathrm{vec}-$ tor triplets at non-polar latitudes. Large-scale magnetospheric contributions were estimated up to degree/order 2; the zonal terms vary with annual and semi-annual periodicity, and terms with degree $n=1$ were modulated with the strength of the magnetospheric ring-current as measured simultaneously by globally distributed geomagnetic observatories. In addition, the observatory data were used to constrain secular variation (they resolve, however, only $2 \%$ of the model).

The model was estimated using Iteratively Reweighted
Least Squares with Huber weights to account for the nonGaussian data error distribution. Achieved rms misfit at non-polar latitudes is $2.9 \mathrm{nT}$ for the scalar intensity and for one of the vector components perpendicular to the magnetic field; the third vector component (rms misfit of $6.4 \mathrm{nT}$ due to attitude noise) was downweighted when estimating the model. More information on the model and its errors can be found in Olsen (2002) and Lowes and Olsen (2004). We believe that the data weighting used in deriving OSVM has some advantages over that used in deriving CM4, and wished to use OSVM as the basis of another IGRF candidate model.

\subsection{Ionospheric leakage: Contributions from iono- spheric and induced currents}

Ionospheric field contributions and their Earth-induced counterparts were considered during the derivation of CM4. This, however, was not the case for OSVM (and most other) geomagnetic field models, and therefore the possibility of "leakage" of the ionospheric field and its induced counterpart into the OSVM internal coefficients has to be considered. We will now investigate the magnitude of this leakage by looking at the difference between the main field parts of OSVM and CM4, and by utilizing the ionospheric field as predicted by CM4 as well as independent models of geomagnetic daily variations. After discussion of the nature of this leakage, we present a scheme of correcting models (like OSVM) that were derived without considering this contribution.

The left part of Fig. 1 shows the magnetic radial component, $B_{r}$, at ground of the difference between the main field parts of OSVM and CM4 at epoch 2000, up to $n=8$. The difference is mainly axially symmetric, and its shape (minima at North pole and southern mid-latitudes; maxima at South pole and northern mid-latitudes) suggests that the coefficient $g_{3}^{0}$ is a main contributor. This is confirmed by the peak at $n=3$ in the Mauersberger-Lowes spectrum of the difference, $R_{n}(\mathrm{OSVM}-\mathrm{CM} 4)$, shown in the left part of Fig. 2.

The main reason for this difference in the main field part between CM4 and OSVM is the different approaches of considering ionospheric and induced contributions. They were co-estimated in CM4, but assumed to be absent in OSVM. Although ionospheric currents are much smaller during the night, their induced counterparts still produce a significant magnetic field contribution, and it must be concluded that the OSVM main field coefficients are contaminated by that effect. This "ionospheric leakage" has been investigated by Lowes and Olsen (2004). They could reasonably estimate the amount of leakage up to $n=8$; in terms of mean-square vector field the leakage amounts to $39 \mathrm{nT}^{2}$.

Ionospheric contributions depend strongly on ionospheric conductivity, which (at non-polar latitudes) is caused by solar radiation. Due to the absence of solar radiation during the night, ionospheric conductivity is reduced by 1-2 orders of magnitude. As a consequence, ionospheric currents, and their magnetic field, are likewise reduced during night-time. To minimize contributions from ionospheric currents, the OSVM had therefore been derived from nightside data, as is common practice in geomagnetic field mod- 


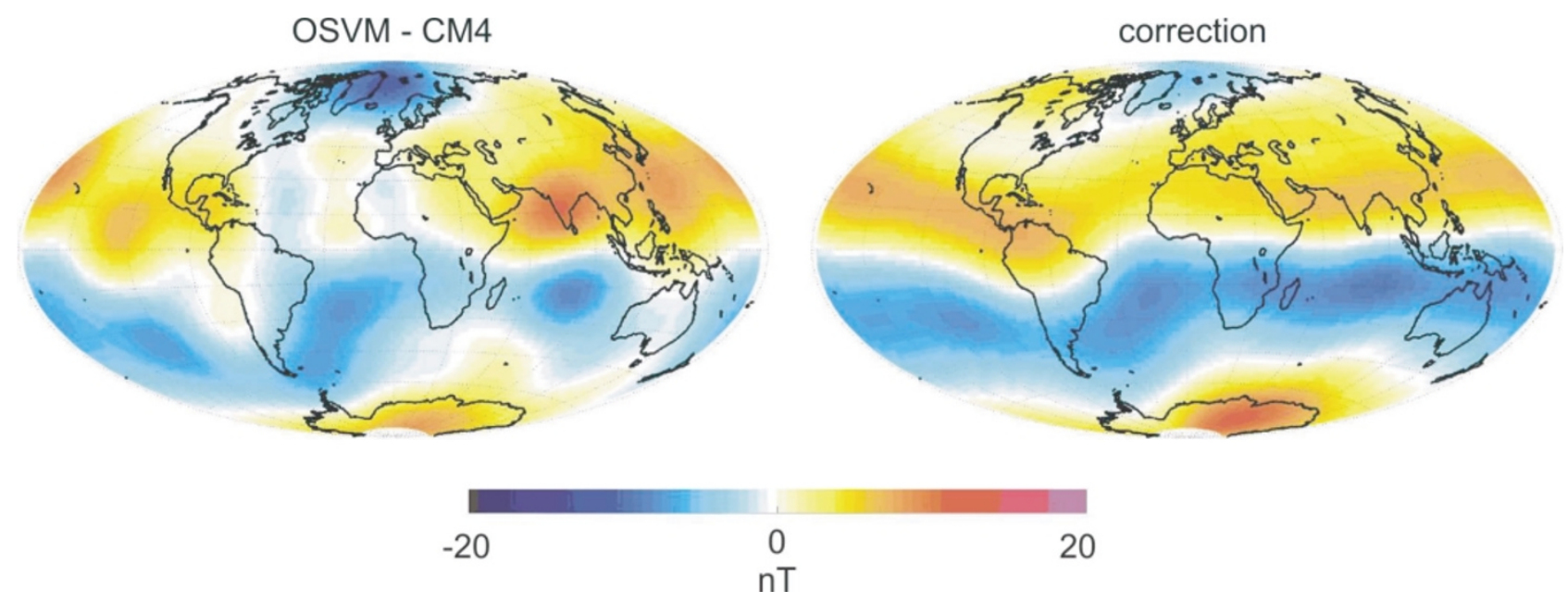

Fig. 1. Maps of $B_{r}$ at ground (Hammer projection), for $n \leq 8$. Left: Difference OSVM minus CM4. Right: Correction for ionospheric leakage.
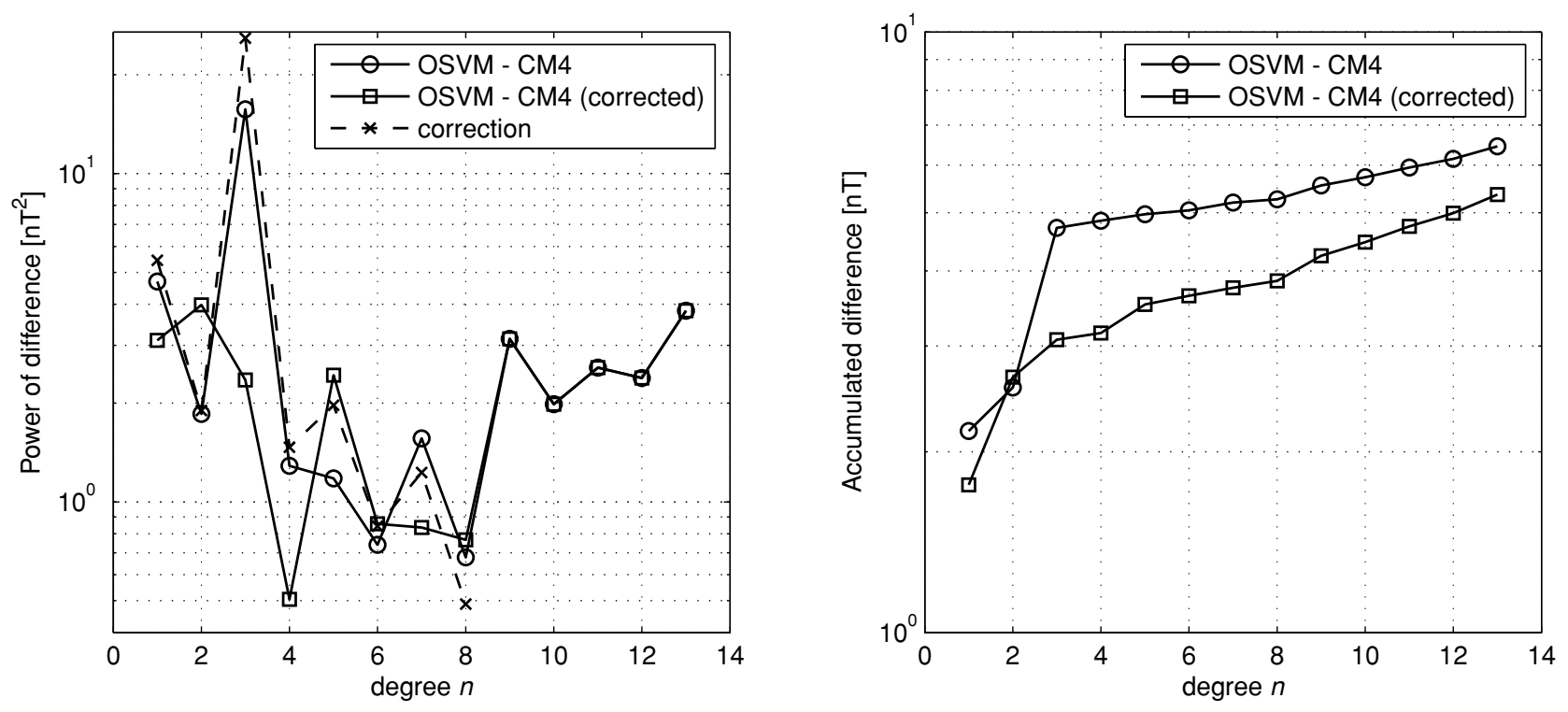

Fig. 2. Left: Mauersberger-Lowes spectrum, $R_{n}(\mathrm{OSVM}-\mathrm{CM} 4)$, of the difference OSVM-CM4, before and after correcting OSVM for ionospheric leakage. The spectrum of the correction is shown by the dashed line. Right: accumulated difference, $\sqrt{\sum_{l=1}^{n} R_{l}(\mathrm{OSVM}-\mathrm{CM} 4)}$.

eling.

However, magnetic field contributions from Earthinduced currents do not vanish during night; they contribute even if night-time ionospheric currents are exactly zero. This was noticed for the first time by Ashour and Price (1965). In addition, ionospheric currents at polar latitudes usually are always present, (i.e. they do not vanish during night since polar ionospheric conductivity is produced by particle precipitation in addition to solar radiation) and therefore their contributions can not be eliminated by selecting night data.

We illustrate the first effect (contribution from induced currents at non-polar latitudes) by using the simple example of Fig. 3. Its upper part shows schematically, for a given location, the ionospheric current (for example $J_{\phi}$ ) for 5 consecutive days. (The magnetic field variation at a fixed location shows a similar form of the time series.) In the simple model considered here, ionospheric currents are assumed to be exactly zero during night-time hours (indicated by the shaded areas) and are maximum at local midnight. Fourier decomposition yields

$$
J^{\mathrm{ext}}(T)=a_{0}+a_{1} \cos T+a_{2} \cos 2 T+a_{3} \cos 3 T+\cdots
$$

where $T$ is local time in radians, with $T=0$ at local midnight. Only cosine terms contribute; sine-terms are zero due to the assumed symmetry around local midnight. Note that $a_{0}$, the daily average, is not zero.

The time-changing parts of the external currents induce secondary, induced, currents, whereas a static external field does not produce any induced field, whatever the conductivity structure. If for simplicity we assume that the Earth consists of an infinitely conducting inner sphere overlaid by an insulator, the time series of the induced field at a given location has the same shape as the external field, but is smaller and shifted in origin such that the daily mean of the induced field becomes zero. (Strictly, we should do this calculation 
daily variation of ionospheric currents, with zero level during night

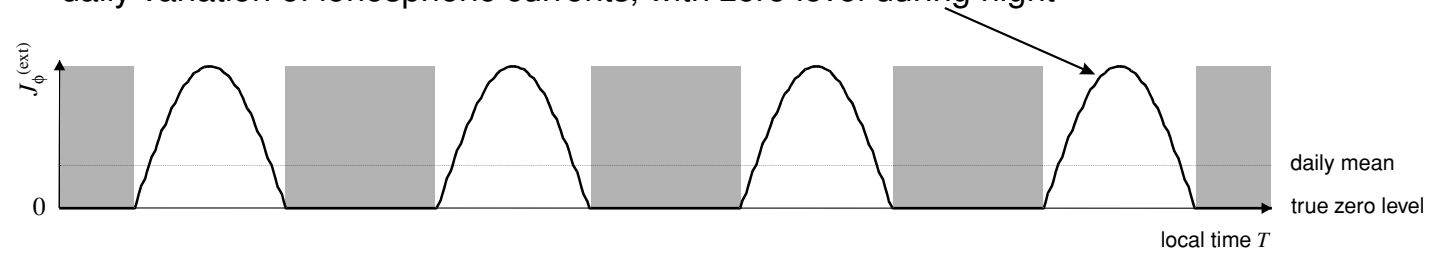

daily variation of induced currents, non-zero level during night!

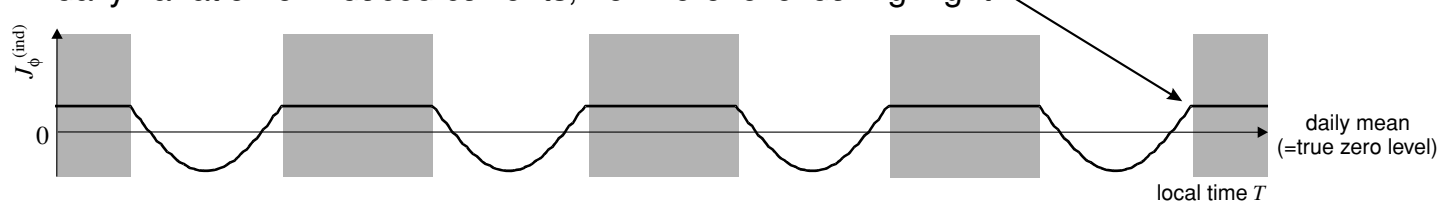

Fig. 3. Sketch of the time-change of ionospheric and induced daily variations.

for the time harmonics associated with each spatial, spherical, harmonic.) For such a simple conductivity model, the transfer function $q$, the ratio between the induced and the external currents, is independent of the frequency of the external signal (except that it is zero for zero-frequency, i.e., for a static field, for any model). Hence the currents induced by the external currents of Eq. (1) follow as

$$
J^{\text {ind }}(T)=q \cdot\left(a_{1} \cos T+a_{2} \cos 2 T+a_{3} \cos 3 T+\cdots\right)
$$

Note the absence of $a_{0}$ in this expression (since $q$ is zero for a static field), which means that the daily mean of the induced currents is zero. Even if the primary, inducing, currents vanish at night, their induced part does not, as shown in the lower part of Fig. 3. As a consequence, induced currents during night are generally non-zero. Their midnight value can be calculated from the expansion coefficients of the external contributions (in this example the coefficients $a_{k}$ ) and the response of the conducting mantle (in this example the constant $q$ ):

$$
J^{\text {ind }}(T=0)=q \cdot \sum_{k=1}^{\infty} a_{k} \neq 0 .
$$

For more realistic mantle conductivity models, for a given spatial harmonic the response $q$ depends on frequency (and is no longer real-i.e. there is a phase shift) and thus is different for different $k$ in the above summation. This means that the shape of the times series of the induced field will be different from that of the inducing field. However, for each harmonic the daily means of the induced currents are always zero, which generally implies non-vanishing induced currents during night-time.

The above example demonstrates that a direct determination of the induced magnetic field contributions during night is possible from the expansion coefficients of geomagnetic daily variations ( $S q$ variations). To illustrate the magnitude of the effect we have used the models of geomagnetic daily variations of Malin (1973) and Winch (1981). Both authors derived spherical harmonic expansions of $S q$ based on hourly mean values from more than 100 worldwide distributed observatories; Malin (1973) for solar maximum conditions (1957-59, mean solar flux $F_{10.7}=200 \cdot 10^{-22}$ $\left.\mathrm{W} /\left(\mathrm{m}^{2} \mathrm{~Hz}\right)\right)$, and Winch (1981) for solar minimum conditions $\left(1964-65, F_{10.7}=65 \cdot 10^{-22} \mathrm{~W} /\left(\mathrm{m}^{2} \mathrm{~Hz}\right)\right)$. The main part of geomagnetic daily variations is a local time phenomena, i.e., the current system moves around the Earth following the movement of the sub-solar point. From the local-time spherical harmonic expansion coefficients of the induced field as determined by Malin (1973) and Winch (1981) we derived the coefficients $g_{n}^{0}, n=1-4$ of induced contributions at midnight. These coefficients are listed in Table 1 and indicate the contamination by induced fields of main field models that are obtained from local midnight data.

Using models of the $S q$ variation that have been derived solely from observatory data (as is the case for the models of Malin (1973) and Winch (1981)) it is not possible to infer the absolute level (cf. the coefficient $a_{0}$ of Eq. (1)) of ionospheric variations; only the induced contribution (which always has zero mean, i.e. does not depend on $a_{0}$ ) can be determined absolutely. Estimation of the absolute ionospheric level, $a_{0}$, requires a joint analysis of observatory and satellite data, as is done in CM4. Rather than using models of the $S q$ variation that are based on observatory data only (with no information on $a_{0}$ ), we use the ionospheric (and corresponding induced) field of CM4 for correcting OSVM for ionospheric leakage.

The left panel of Fig. 4 shows a snapshot of the magnetic radial component at ground due to ionospheric (top) and induced (bottom) currents, as given by CM4, for March, 21 $2000,12: 00$ UT and a solar flux value of $F_{10.7}=150 \cdot 10^{-22}$ $\mathrm{W} /\left(\mathrm{m}^{2} \mathrm{~Hz}\right)$. The magnetic field of the $S q$ current system and its induced counterpart is clearly seen, with up to $40 \mathrm{nT}$ in $B_{r}$ at mid-latitudes at local noon for the ionospheric and 10-15 nT for the induced field.

The right panel shows the corresponding magnetic field at each point for local midnight, $T=0$; note that this is not a snapshot in time, since local midnight does not occur simultaneously all over the globe. As expected from the much reduced ionospheric conductivity at middle and low latitudes, the ionospheric magnetic field (upper panel) is close to zero at those latitudes, but reaches values of up to $15 \mathrm{nT}$ at polar latitudes (since the polar ionospheric conduc- 
Table 1. Contribution of ionospheric and induced fields to the main field coefficients $g_{n}^{0}, n=1-4$. The values for $1957-59$ and $1964-65$ are derived from the spherical harmonic expansion of geomagnetic daily variations done by Malin (1973) and Winch (1981), respectively. Contributions as given by CM4 are for solar maximum.

\begin{tabular}{l|r|rrrr} 
& \multicolumn{1}{|c|}{$\begin{array}{c}F_{10.7} \\
{\left[\mathrm{~W} /\left(\mathrm{m}^{2} \mathrm{~Hz}\right)\right]}\end{array}$} & $\begin{array}{c}g_{1}^{0} \\
{[\mathrm{nT}]}\end{array}$ & $\begin{array}{c}g_{2}^{0} \\
{[\mathrm{nT}]}\end{array}$ & $\begin{array}{c}g_{3}^{0} \\
{[\mathrm{nT}]}\end{array}$ & $\begin{array}{c}g_{4}^{0} \\
{[\mathrm{nT}]}\end{array}$ \\
\hline induced, solar maximum (1957-59) & $200 \cdot 10^{-22}$ & 3.31 & 0.63 & -2.77 & -0.26 \\
induced, solar minimum (1964-65) & $65 \cdot 10^{-22}$ & 2.42 & 0.17 & -0.88 & -0.02 \\
induced, from CM4 & $150 \cdot 10^{-22}$ & 3.26 & -0.19 & -1.40 & 0.07 \\
ionospheric, from CM4 & $150 \cdot 10^{-22}$ & -2.08 & 0.41 & -1.07 & -0.12 \\
sum, from CM4 & $150 \cdot 10^{-22}$ & 1.18 & 0.22 & -2.47 & -0.05 \\
\hline
\end{tabular}
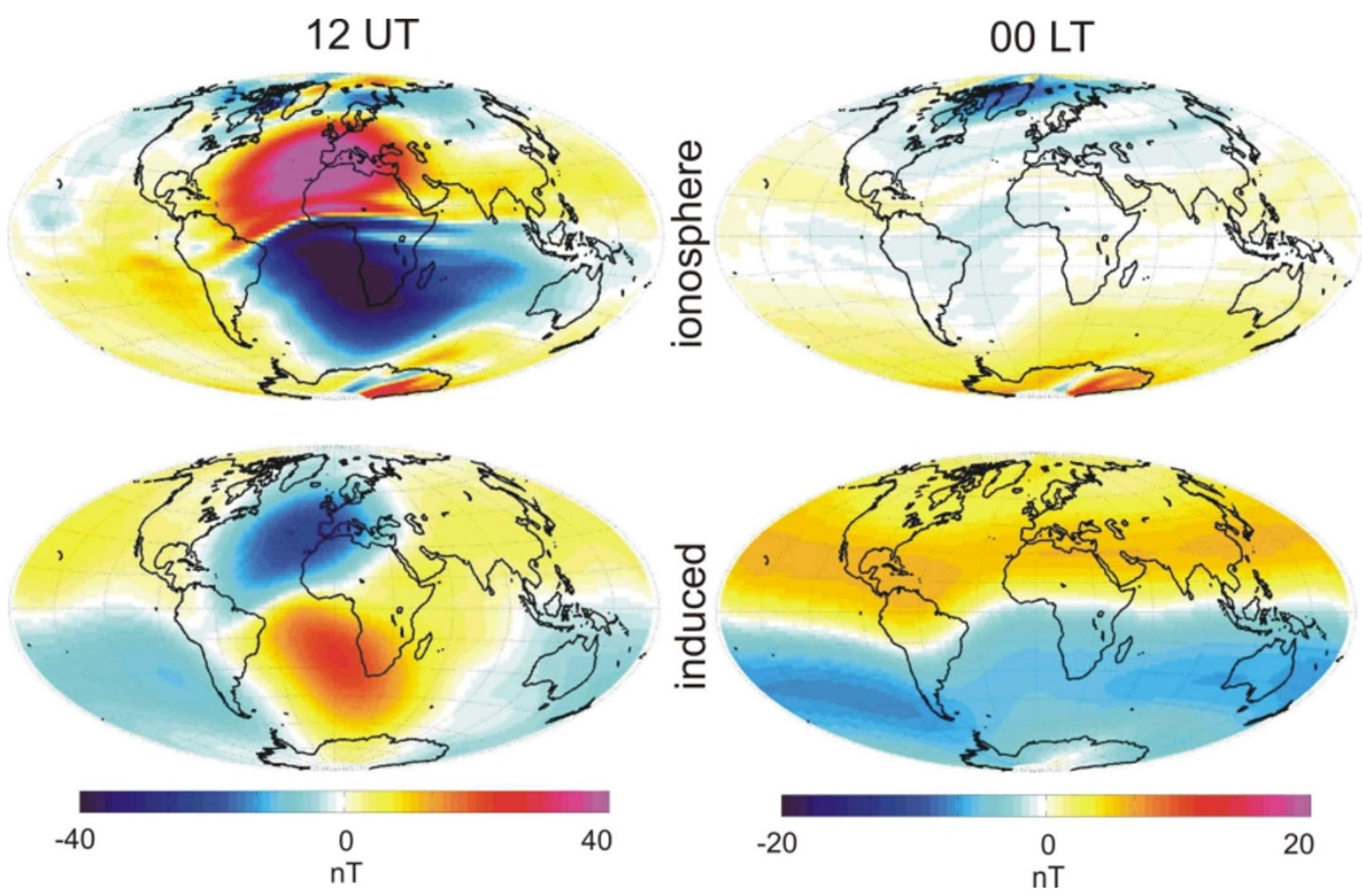

Fig. 4. Magnetic radial component for March 21, 2000 at ground due to ionospheric (top) and induced (bottom) currents, as predicted by CM4. The left part shows a snapshot for 12 UT; the right panel shows the field contributions at the respective local midnight (00 LT).

tivity does not vanish during night). However, induced contributions (lower panel) are significant at all latitudes and all local times. Note that the polar ionospheric field does not contribute significantly to the induced field. This is probably due to the fact that it is quasi-stationary (at least during quiet times). This result should however be taken with care, since it might be a result of the model parameterization of CM4 (which is not optimal for describing the variability of polar current systems).

The midnight fields are mainly independent of longitude and antisymmetric with respect to the equator, and therefore dominated by the odd degree zonal coefficients $g_{1}^{0}, g_{3}^{0}, \ldots$. Table 1 lists $g_{n}^{0}$ for $n=1-4$ produced by the ionospheric, respectively induced, part of CM4. There is reasonable agreement between the induced field contribution as predicted by CM4 and those derived from the independent models of Malin (1973) and Winch (1981). Note that the sign of $g_{1}^{0}$ is reversed between the ionospheric and induced fields, and hence these fields tend to cancel each other in $g_{1}^{0}$, which is the reason for the dominance of $g_{3}^{0}=-2.47 \mathrm{nT}$ (compared to $g_{1}^{0}=1.18 \mathrm{nT}$ ) if ionospheric and induced contributions are combined, and explains the peak at $n=3$ in the left panel of Fig. 2.

These numbers would represent the ionospheric leakage into the OSVM model if all data were taken exactly at local midnight, and at that solar flux. However, the actual data window spans local times between 19:00 and 07:00, and a range of solar flux. It was therefore decided to estimate ionospheric leakage indirectly, as described in more detail in Lowes and Olsen (2004). The ionospheric and induced field contributions, as predicted by CM4, were calculated for each data point that went into the OSVM modeling, and the effect on the output coefficients noted. The corresponding radial magnetic field correction is shown in the right panel of Fig. 1 (which is close, but not equal, to the sum of ionospheric and induced fields shown in the right panel of Fig. 4). 


\subsection{The OSVM candidate for DGRF 2000}

The candidate for DGRF 2000 (called DGRF2000OSVM) is a $n_{\max }=13$ truncation of the OSVM internal field coefficients for epoch 2000, corrected for ionospheric leakage and rounded to nearest $0.1 \mathrm{nT}$. In detail:

1) To produce the candidate model we have subtracted from the OSVM coefficients the estimate of the $n \leq 8$ ionospheric leakage, as described in the previous section. This reduces the accumulated difference between CM4 and OSVM, $\sqrt{\sum_{l=1}^{n} R_{l}(\mathrm{OSVM}-\mathrm{CM} 4)}$, shown in the right panel of Fig. 2, for $n>2$. This accumulated difference may be regarded as an estimate of the accumulated model error, which is about $5 \mathrm{nT}$ for degrees up to $n=12$ after applying the correction. Without correction, a $5 \mathrm{nT}$ accumulated error is already reached at degree $n=5$.

2) A full model covariance matrix has been estimated for the OSVM coefficients. However, Lowes and Olsen (2004) have shown that, because the modeling ignored the serial correlation of the residual (mainly magnetospheric) noise, the variances quoted for the OSVM sectoral $(n=m)$ and near-sectoral coefficients were too small. Similarly, leakage of the day-to-day variations of the ionospheric field meant that the quoted variances for the axial and near-axial coefficients were also too small. Conversely, for other coefficients the quoted variances were too large. They estimated a smoothly varying correction factor, which has been applied to the original OSVM variance estimates.

3) The leakage subtracted in step 1) is only an approximation. We estimate (somewhat arbitrarily) that the correction is accurate to about $12 \%$ for each coefficient, corresponding to a (pseudo-random) variance equal to about $25 \%$ of the square of the coefficient. We have added this variance contribution to the variance produced by step 2) above.

4) The covariances, a $195 \times 195$ matrix are then estimated (fairly arbitrarily) using the final variance estimates (combination of steps 2) and 3)). It is available from http://www.spacecenter.dk/projects/oersted/models.
The original OSVM correlation matrix and the original OSVM covariance matrix, both truncated to $n \leq 13$, are also available at that site.

\section{Conclusions}

We described the extraction of three candidate models (one for DGRF 1995, and two for DGRF 2000) for the 9th generation of the IGRF. These models are based on the CM4 (Sabaka et al., 2004), respectively the OSVM (Olsen, 2002; Lowes and Olsen, 2004), as parent models. However, since the low-degree zonal coefficients of the main field part of OSVM are contaminated by ionospheric and induced contributions, we derived a scheme to correct for this contamination, which affects (by 1-2 nT) mainly the coefficients $g_{1}^{0}$ and $g_{3}^{0}$.

We believe that most, if not all, models that have been derived primarily from local night-time data suffer from such an ionospheric leakage. The presented correction scheme could also be be applied to approximately correct other models.

\section{References}

Ashour, A. A. and A. T. Price, Night-time earth currents associated with the daily magnetic variations, Geophys. J. R. Astron. Soc., 10, 1-15, 1965

Lowes, F. J. and N. Olsen, A more realistic estimate of the variances and systematic errors in spherical harmonic geomagnetic field models, Geophys. J. Int., 157, 1027-1044, 2004.

Malin, S. R. C., Worldwide distribution of geomagnetic tides, Phil. Trans. Roy. Soc. London, A 274, 551-594, 1973.

Mandea, M., Candidate main-field models for producing the 9th generation IGRF, Earth Planets Space, 57, this issue, 1183-1189, 2005.

Olsen, N., A model of the geomagnetic field and its secular variation for epoch 2000 estimated from Ørsted data, Geophys. J. Int., 149, 454-462, 2002

Sabaka, T. J., N. Olsen, and R. A. Langel, A comprehensive model of the quiet-time near-Earth magnetic field: Phase 3, Geophys. J. Int., 151, 32 $68,2002$.

Sabaka, T. J., N. Olsen, and M. Purucker, Extending comprehensive models of the Earth's magnetic field with Ørsted and CHAMP data, Geophys. J. Int., 159, 521-547, doi:10.1111/j.1365-246X.2004.02,421.x, 2004

Winch, D. E., Spherical harmonic analysis of geomagnetic tides, Phil. Trans. Roy. Soc. London, A 303, 1-104, 1981.

N. Olsen (e-mail: nio@spacecenter.dk), F. Lowes, and T. J. Sabaka 